\title{
HARPO - TPC for High Energy Astrophysics and Polarimetry from the MeV to the GeV
}

\section{Philippe GROS*}

LLR, Ecole Polytechnique, CNRS/IN2P3 (FR)

E-mail: philippe.grosallr.in2p3.fr

D. Bernard, Ph. Bruel, M. Frotin, Y. Geerebaert, B. Giebels, D. Horan, P. Poilleux, I. Semeniouk, S. Wang

LLR, Ecole Polytechnique, CNRS/IN2P3 (FR)

\section{S. Anvar, D. Attié, P. Colas, A. Delbart, P. Sizun}

Irfu, CEA Saclay (FR)

D. Götz

AIM, CEA/DSM-CNRS-Université Paris Diderot,

Irfu/Service d'Astrophysique, CEA Saclay (FR)

\begin{abstract}
Observation of high-energy sources requires gamma-ray telescopes aboard balloons or satellites to study non thermal phenomena (black holes, neutron stars, active galactic nuclei, supernovae, supernova remnants, and gamma-ray bursts). In recent years, $R \& D$ has been mainly active to improve the sensitivity required for polarimetry. In this context, a concept of a Time Projection Chamber (TPC) was proposed as an active target and pair production imager with a high angular resolution and background reduction capabilities.

After introducing the HARPO TPC and its potential as gamma-ray telescope, we present the characterization of the TPC readout plane which provides gas electron amplification within a microstructure composed of the association of a Micromegas and Gas Electron Multiplier. Recent results using cosmic-ray events are shown and finally the beam test, scheduled this year, with polarized photon at $\mathrm{MeV}$ energy will be discussed.
\end{abstract}

Technology and Instrumentation in Particle Physics 2014,

2-6 June, 2014

Amsterdam, the Netherlands

\footnotetext{
* Speaker.
} 


\section{Introduction}

Cosmic gamma-rays are important tools to study non-thermal astrophysical phenomena. Their polarisation could give a lot of insight on astronomical objects like blazars [4] and Gamma-Ray Bursts (GRB) [5]. They even have the potential to test fundamental physics question like Lorentz Invariance Violation (LIV) [6].

Gamma-ray telescopes have improved in sensitivity and resolution from COS-B [2] to FermiLAT [3], using the concept of photon conversion into an electron-positron pair. The performance drops, however, for photons below $100 \mathrm{MeV}$, where the background becomes problematic and the angular resolution becomes limited by multiple scattering. Meanwhile, telescopes using Compton scattering (like COMPTEL [1]) are still far from reaching the same level of accuracy for energies above $1 \mathrm{MeV}$.

We propose a gaseous detector, based on the Time Projection Chamber (TPC) concept, to cover this gap between a few $\mathrm{MeV}$ and $100 \mathrm{MeV}$ (and up to the $\mathrm{GeV}$ ) in gamma-ray detection. The TPC would have the added advantage of providing information on the photon polarisation. We will first review the performance potential of such a detector, and then present the HARPO (Hermetic Argon POlarimeter) TPC demonstrator. After showing results from characterisation tests, we will present the upcoming beam test and future developments.

\section{A Time Projection Chamber for gamma-ray detection and polarimetry}

The angular resolution and polarimetry potential of a TPC has been studied in detail in [7] and [8]. The angular resolution for pair production is limited by multiple scattering of the electrons in the gas. Fig. 1 shows the resolution limit in 10 bar argon. Besides, most conversions will happen in the field of a nucleus, and its recoil momentum is impossible to measure. This puts a strong limit on the resolution at low energies $(<100 \mathrm{MeV})$. The final resolution can still be an improvement up to an order of magnitude compared to the current Fermi results.

The theoretical studies and simulations in [7] show that this setup can be used to get polarisation information on the incoming photons. The results show that a TPC would have a good potential for specific astronomical studies of gamma-ray polarimetry. These results need however to be validated experimentally.

\section{The HARPO TPC demonstrator}

A TPC prototype, named HARPO (Hermetic ARgon POlarimeter) was conceived and built to demonstrate the expected performance in a test beam environment. The HARPO TPC [10] is a $30 \mathrm{~cm}$ cubic drift volume, set in an aluminum vessel able to withstand more the 5 bar pressure. It is equipped with an amplification plane made of a stack of one micromegas [12] and two GEM [11] sheets. The electron readout is made by two series of 288 copper strips with $1 \mathrm{~mm}$ pitch, with perpendicular orientation. The signal is then treated and digitised with AFTER chips [15]. The system is also equipped with six scintillator plates used for triggering. A layout of the detector is shown in Fig. 2.

It was decided to stack GEMs on top of the micromegas to be able to reach sufficient amplification at high pressure ( $>2$ bar). We performed two different tests to characterise the amplification 


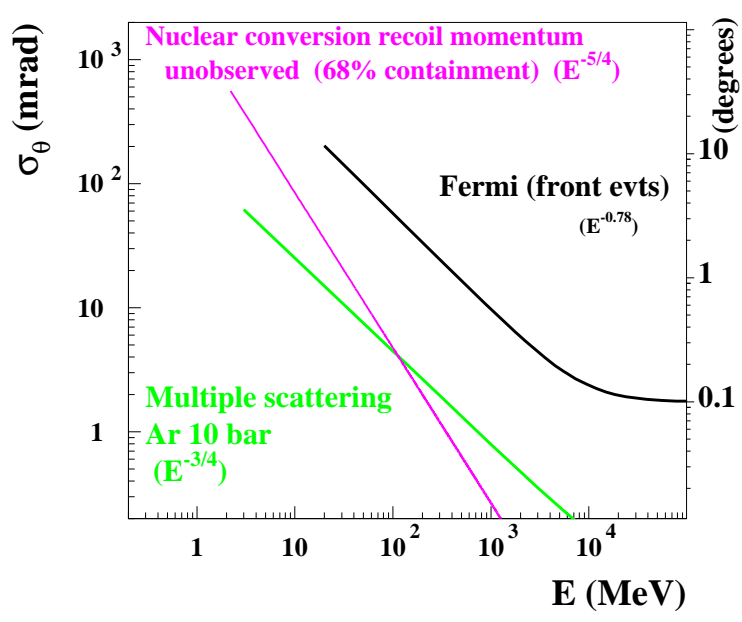

Figure 1: Angular resolution potential for a TPC with 10 bar argon. At high energies ( $>100 \mathrm{MeV})$, it is limited by the multiple scattering of the electrons in the gas. At lower energies, it is constrained by the unknown recoil momentum of the nucleus. This limit can be overcome by looking at triplet conversion, but they are rarer and more difficult to reconstruct.

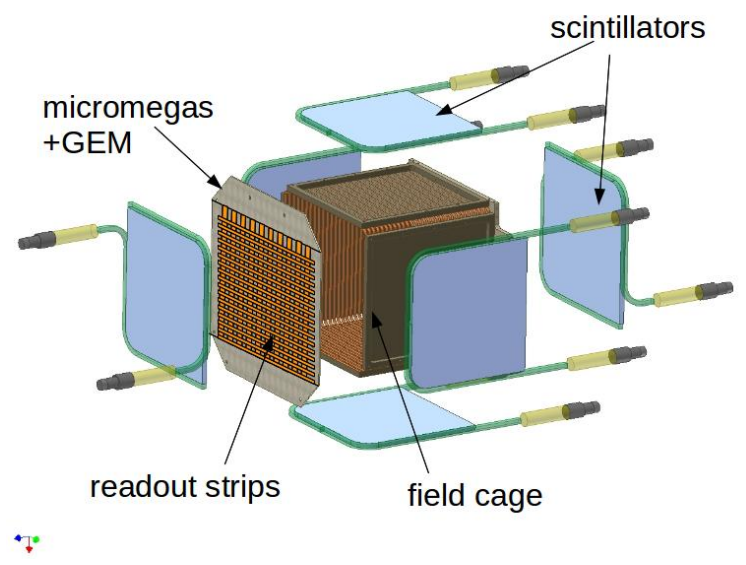

Figure 2: sketch of HARPO. A cubic TPC in the center, with a readout plane of micromegas and GEM on the left. Six scintillator plates, each equipped with a wavelength shifter and two photomultipliers, are used for trigger. The system fits in an aluminum cylinder able to withstand up to 5 bar pressure.

system. The system with micromegas and GEMs was first tested in a small test box, at atmospheric pressure, with an ${ }^{55} \mathrm{Fe}$ radioactive source. Then, it was installed in the TPC, and further characterisation was done at different pressures using cosmic rays.

\subsection{Test of the amplification with radioactive sources}

A first test of the amplification system was done in a simple test box where one or two GEMs were stacked on top of the micromegas plane. The readout strips were grounded.The GEM layer were separated by $2 \mathrm{~mm}$ spacers, so that the layout was as described in Fig. 3. We then read the signal on the micromegas mesh through a readout chain composed of a pre-amplifier Ortec 142b, 
an amplifier and shaper Ortec 572, and a multichannel analyser Amptek 8000a. The box was filled with a flowing Ar:isobutane (95:5) gas mixture at atmospheric pressure.
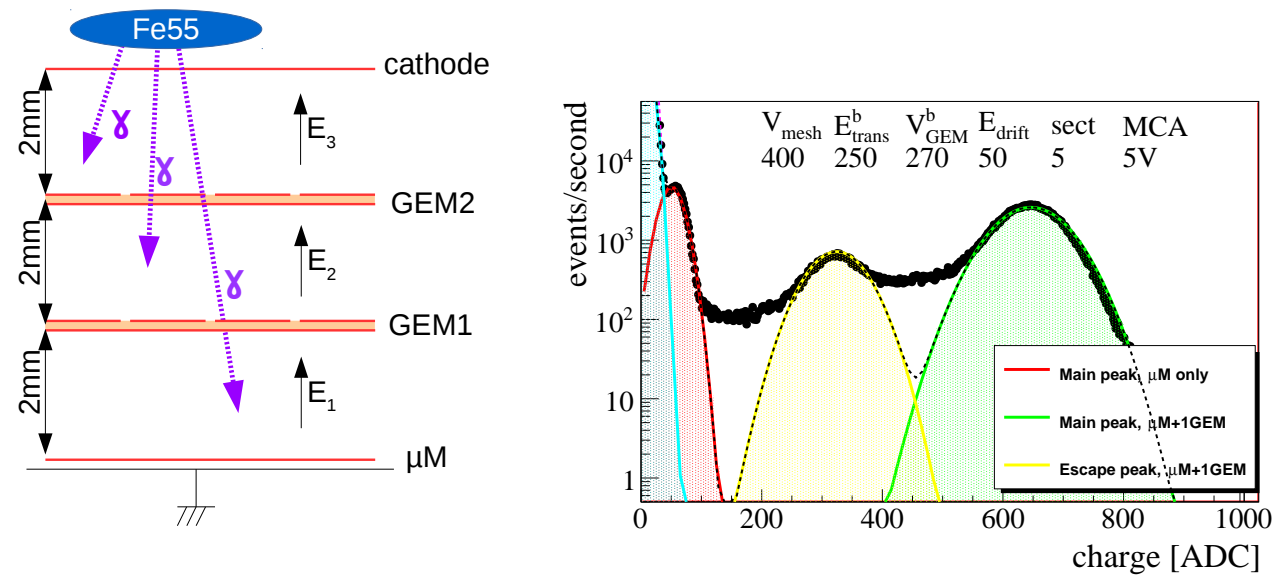

Figure 3: Left: layout of the test setup, with one micromegas and two GEMs. By applying a null or inverted voltage to the top GEM, we only observe conversion in the two lower regions. Right: example of measured ${ }^{55} \mathrm{Fe}$ spectrum with amplification with one micromegas and one GEM. We see the main peak (5.9 keV) after amplification with only the micromegas (in red) or with both micromegas and GEM (in green). The escape peak is only visible with full amplification (in yellow).

Measurements were performed using ${ }^{55} \mathrm{Fe}$ sources for different field configurations. The 2.7 and $5.9 \mathrm{keV}$ signals of the X-ray conversion in argon (with and without escaping photon) could be observed. Since the conversion could happen in different regions between the amplification layers, we observe the signal with different amplification. If the conversion takes place between the micromegas and the first GEM, the electrons are only amplified in the micromegas. If it takes place above the first GEM, they will be amplified twice. Fig. 3 shows the resulting spectrum in such a situation. In that figure, we see the two peaks (main and escape) from the full micromegas+GEM amplification, and the main peak with only micromegas amplification (the escape peak is lost in the noise). The ratio between the main peaks with and without GEM amplification give an absolute measurement of the GEM gain.

We can describe the effective gain of a Micro-Pattern Gas Detector (MPGD: GEM or micromegas) with three components:

proper gain $g$ is the actual amplification of the electrons. It depends on the field inside the holes, and therefore on the applied voltage $V_{G E M}$. The dependence is typically exponential

collection efficiency $\mathscr{C}$ is the probability for an electron coming from the drift volume to reach the amplification region in the hole. It depends on the drift field $E_{\text {drift }}$ above the MPGD and the diffusion in the gas. It should more precisely depend on $E_{d r i f t} / V_{M P G D}$ if we only take into account field geometry, but we will neglect that extra dependence here, because the variations of $V_{M P G D}$ are relatively small.

extraction efficiency $\mathscr{E}$ is the probability for an electron in the hole to reach the transfer region below the GEM. It depends mostly on the transfer field $E_{\text {trans }}$. Here we neglect again the 
dependence with $V_{G E M}$. This of course does not apply for micromegas.

We obtain the following expression for the effective gain of the stack of micromegas and two GEMs (where $E_{1}, E_{2}$ and $E_{3}$ are defined in Fig. 3):

$$
\begin{aligned}
g_{\text {tot }}^{\text {eff }} \propto & g_{\text {micromegas }}\left(V_{\text {mesh }}\right) \times \mathscr{C}_{\text {micromegas }}\left(E_{1}\right) \\
& \times g_{G E M}\left(V_{G E M 1}\right) \times \mathscr{C}_{G E M}\left(E_{2}\right) \times \mathscr{E}_{G E M}\left(E_{1}\right) \\
& \times g_{G E M}\left(V_{G E M 2}\right) \times \mathscr{C}_{G E M}\left(E_{3}\right) \times \mathscr{E}_{G E M}\left(E_{2}\right)
\end{aligned}
$$

We see that the total effective gain shows complex dependencies, but when we extract the effective gain of the individual GEMs as explained above, we can evaluate the different components. The result is shown in Fig. 4, with arbitrary normalisations. On the right plot, we see that the effective gain depends exponentially on the applied voltage, as expected from a simple description of the amplification [13]. On the left plot, we see that the collection efficiency is $100 \%$ up to $500 \mathrm{~V} / \mathrm{cm}$ for the micromegas and $1000 \mathrm{~V} / \mathrm{cm}$ for the GEM. We also see that the extraction efficiency does not reach any saturation, so that it is difficult to normalise it properly. The measured extraction efficiency might be modified by a dependence of the proper gain with $E_{\text {trans }}$ (as the length of the amplification region might increase with $\left.E_{\text {trans }}\right)$.
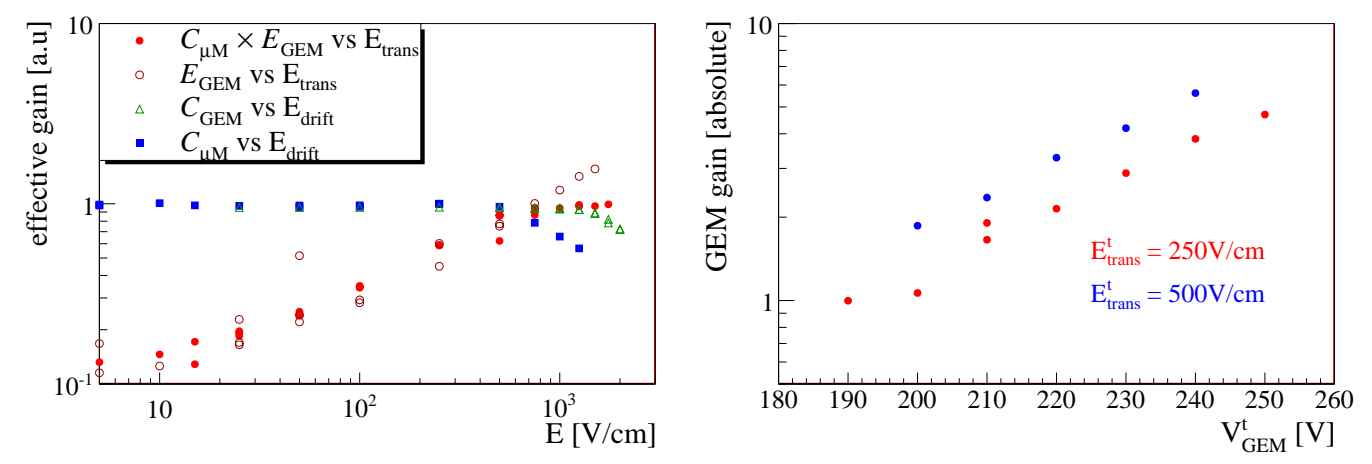

Figure 4: Micromegas and GEM gain measured in a test box with ${ }^{55} \mathrm{Fe}$ source. Left: dependence with the field above $\left(E_{d r i f t}\right)$ and below $\left(E_{\text {trans }}\right)$ the amplification region. The results are arbitrarily normalised. They can be interpreted as the collection $\mathscr{C}$ and extraction $\mathscr{E}$ efficiencies of the micromegas and GEM. Right: absolute effective gain of GEMs as a function of the applied voltage. An exponential law describes it down to values very close to unity. Measurement of low values $(<2)$ are limited by the energy resolution. Measurement of high values $(>5)$ are limited by the dynamic range of the electronics. Extrapolation shows that we operated the GEMs at gains up to 25 .

\subsection{Tests of amplification with cosmic rays}

The characterisation of the amplification system was completed by some tests in the HARPO TPC, using cosmic rays. The TPC was oriented so that cosmic rays would traverse it along the $\mathrm{z}$-axis. In that configuration, the average charge deposited in the TPC in approximately uniform along that axis, as shown in Fig. 5 (left). By assuming that most of the tracks come from minimum ionising particles (MIPs), we can easily estimate the effective gain of the amplification in the TPC. Fig. 5 (right) shows that gain for different values of the gas pressure in the TPC, as a function of the voltage on one of the GEMs. We observe the same behaviour as in the test box. 

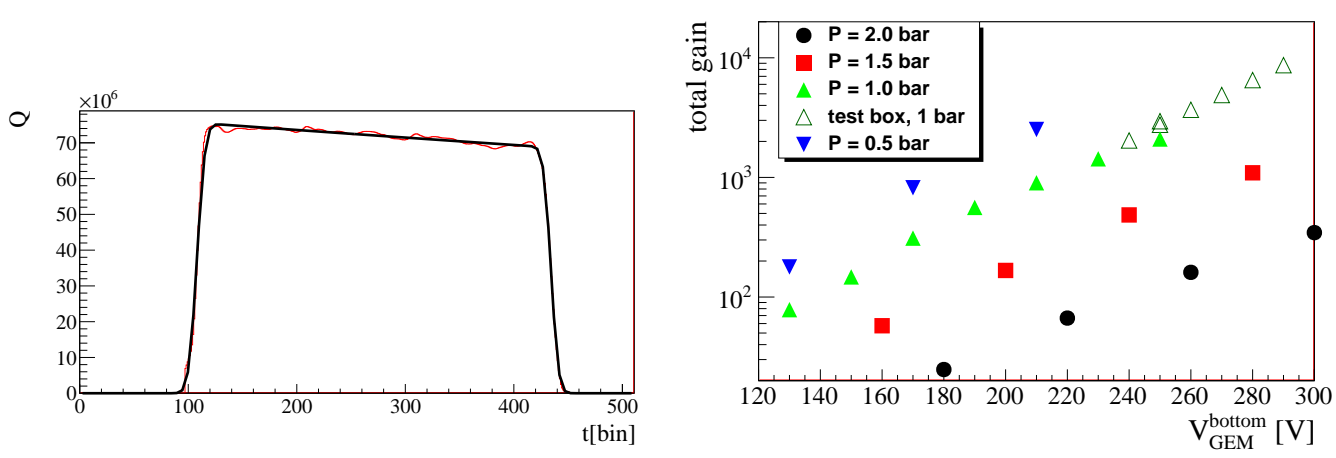

Figure 5: Left: average energy deposited at each time bin for cosmic tracks along the $\mathrm{z}$-axis. This measurement can be used to extract the drift velocity (from the length of the distribution), the effective gain on the readout plane (from the surface) and the electron absorption in the drift volume (from the slope). Right: effective gain measured from cosmic rays in the TPC, for different gas pressures. The results at 1 bar are consistent with those obtained with radioactive sources.

\section{Conclusions and perspectives}

We proposed a TPC to cover the performance gap in cosmic gamma-ray detection in the $\mathrm{MeV}$ $\mathrm{GeV}$ range. A demonstrator was built with micromegas and GEM amplification system. The first characterisation tests give interesting results on the behaviour of MPGDs in different field configurations. Further studies of the test data, and comparison with simulations may help finding an optimal configuration, especially at higher pressure.

The demonstrator is now ready for data taking in a polarised photon beam. A beam campaign is scheduled for November 2014 at the NewSUBARU [14] accelerator. This will allow to check the expected angular resolution and sensitivity to polarisation for photons from 4 to $76 \mathrm{MeV}$, and get benchmarks for a simulation of the detector.

Further development is ongoing to meet the constraints of a space environment [9]. Microbulk micromegas [17] are investigated for a robust amplification at high pressure. The trigger is one of the main issues, and the developments in self-triggered electronics like AGET [16] give interesting options.

This work is funded by the P2IO LabEx (ANR-10-LABX-0038) in the framework "Investissements d'Avenir" (ANR-11-IDEX-0003-01) managed by the French National Research Agency (ANR), and directly by the ANR (ANR-13-BS05-0002).

\section{References}

[1] V. Schönfelder, Lessons learnt from COMPTEL for future telescopes, New Astr. Rev. 48 (2004) 193

[2] H. Bloemen, Ann. Rev. Astron. Astrophys. 27 (1989) 469.

[3] M. Ackermann et al. [Fermi-LAT Collaboration], The Fermi Large Area Telescope On Orbit: Event Classification, Instrument Response Functions, and Calibration, Astrophys. J. Suppl. 203, 4 (2012) [arXiv:1206.1896 [astro-ph.IM]].

[4] H. Zhang and M. Böttcher, X-Ray and Gamma-Ray Polarization in Leptonic and Hadronic Jet Models of Blazars, Astrophys. J. 774, 18 (2013) [arXiv:1307.4187 [astro-ph.HE]]. 
[5] D. Eichler, A. Levinson, Polarization of GRB via scattering off a relativistic sheath, Astrophys. J. 596, L147 (2003). [astro-ph/0306360].

[6] T. Jacobson, S. Liberati and D. Mattingly, Lorentz violation at high energy: Concepts, phenomena, and astrophysical constraints, Ann. Phys., 321 (2006) 150

[7] D. Bernard, TPC in gamma-ray astronomy above pair-creation threshold, Nucl. Instrum. Meth. A 701, 225 (2013) [Erratum-ibid. A 713, 76 (2013)] [arXiv:1211.1534 [astro-ph.IM]].

[8] D. Bernard, Polarimetry of cosmic gamma-ray sources above $e^{+} e^{-}$pair creation threshold, Nucl. Instrum. Meth. A 729, 765 (2013) [arXiv:1307.3892 [astro-ph.IM]].

[9] D. Bernard, P. Bruel, M. Frotin, Y. Geerebaert, B. Giebels, P. Gros, D. Horan and P. Poilleux et al., Presented at SPIE2014. To be published. arXiv:1406.4830 [astro-ph.IM].

[10] D. Bernard, HARPO - A Gaseous TPC for High Angular Resolution Gamma-Ray Astronomy and Polarimetry from the MeV to the TeV, Nucl. Instrum. Meth. A 718, 395 (2013)

[11] F. Sauli, GEM: A new concept for electron amplification in gas detectors, Nucl. Instrum. Meth. A 386, 531 (1997).

[12] Y. Giomataris, P. Rebourgeard, J. P. Robert and G. Charpak, MICROMEGAS: A high-granularity position-sensitive gaseous detector for high particle-flux environments, Nucl. Instrum. Meth. A 376, 29 (1996).

[13] T. Aoyama Generalized gas gain formula for proportional counters, Nucl. Instrum. Meth. A 234, 125 (1985).

[14] S Amano et al., Several-MeV $\gamma$-ray generation at NewSUBARU by laser Compton backscattering, Nucl. Instrum. Meth. A 6022009337.

NewSUBARU is a facility operated by LASTI, the Laboratory of Advanced Science and Technology for Industry of University of Hyôgo, Japan.

[15] P. Baron, D. Calvet, E. Delagnes et al., AFTER, an ASIC for the readout of the large T2K time projection chambers, IEEE Trans. Nucl. Sci. 55, 1744 (2008).

[16] S. Anvar, P. Baron et al., AGET, the GET front-end ASIC, for the readout of the Time Projection Chambers used in nuclear physic experiments, Nuclear Science Symposium and Medical Imaging Conference (NSS/MIC), 2011 IEEE 745 - 749.

[17] D. Attié et al., Towards smaller gap microbulks, JINST 9, C04013 (2014). 